\title{
Challenging two-staged percutaneous coronary intervention in multivessel coronary artery disease with a high SYNTAX score: feasible, yet complicated
}

Aleksandra Gąsecka, Aleksandra Bury, Małgorzata Mierzejewska, Arkadiusz Pietrasik

$1^{\text {st }}$ Chair and Department of Cardiology, Medical University of Warsaw, Warsaw, Poland

Submitted: 19 March 2021

Accepted: 2 May 2021

Arch Med Sci Atheroscler Dis 2021; 6: e120-e122

DOI: https://doi.org/10.5114/amsad.2021.107812

Copyright $@ 2021$ Termedia \& Banach

Coronary artery disease (CAD) revascularization either by percutaneous coronary intervention $(\mathrm{PCl})$ or coronary artery bypass grafting (CABG) improves the prognosis in patients with chronic coronary syndrome [1]. Whereas $\mathrm{PCl}$ is the gold standard for single-vessel disease, the choice of treatment for multivessel disease (MVD) is more challenging.

A 71-year-old male patient with MVD was admitted to the cardiology department with unstable angina. Electrocardiography showed atrial fibrillation with ventricular rate $70 / \mathrm{min}$, no conduction disturbances and negative $\mathrm{T}$ waves in I, aVL and V4-V6 leads. Echocardiography demonstrated an enlarged left atrium (area $42 \mathrm{~cm}^{2}$ ), left ventricle hypertrophy (interventricular septum thickness $1.5 \mathrm{~cm}$, posterior wall thickness $1.4 \mathrm{~cm}$ ), no wall motion abnormalities with the left ventricle ejection fraction $60 \%$, and mild regurgitation of the mitral, tricuspid and aortic valves. Coronary angiography showed $70 \%$ stenosis of the left main coronary artery (LMCA), diffuse significant lesions in the left anterior descending artery (LAD), $60-70 \%$ stenosis of the intermediate branch (IM), chronic total occlusion (CTO) of the circumflex artery in the middle segment with collateral circulation from the LAD and right coronary artery (RCA) to the distal segment, and diffuse lesions in the RCA with a fractional flow reserve of 0.63 (Figures $1 \mathrm{~A}, \mathrm{C}$ ). Considering the high anatomical complexity of the lesions (SYNTAX score 34), the Heart Team opted for surgical revascularization, which the patient refused. Regarding persistent ischemic symptoms, an attempt of percutaneous treatment was made.

During the first $\mathrm{PCl}$, femoral access was chosen due to the high lesion complexity. Initial wire manipulations led to thrombus formation in the LMCA, with TIMI 1 flow in the LAD. The patient required immediate dobutamine support and abciximab administration. Emergency PCI of the LMCA towards LAD was performed with everolimus-eluting stent implantation (EES; $3.5 \times 23 \mathrm{~mm})$. Subsequently, two overlapping EES $(3.0 \times 33 \mathrm{~mm}$; $2.5 \times 33 \mathrm{~mm}$ ) were implanted in the proximal and middle segments of the LAD (Figure $1 \mathrm{~B}$ ). An attempt was made to insert the guidewire into the proximal segment of the chronically obstructed circumflex artery. This was not feasible due to the origin angle close to $90^{\circ}$, requiring the use of a $90^{\circ}$ microcatheter, which was not available.

The patient remained hypotonic and required dobutamine support $(5-10 \mu \mathrm{g} / \mathrm{kg} / \mathrm{min})$ for $24 \mathrm{~h}$ after the procedure. Despite pre-hydration, he experienced transient acute kidney injury. In addition, pseudoaneurysm

\author{
Corresponding author: \\ Aleksandra Gąsecka \\ $1^{\text {st }}$ Chair and Department \\ of Cardiology \\ Medical University \\ of Warsaw \\ Warsaw, Poland \\ Phone: +48518 343599 \\ E-mail: aleksandra.gasecka@ \\ wum.edu.pl
}



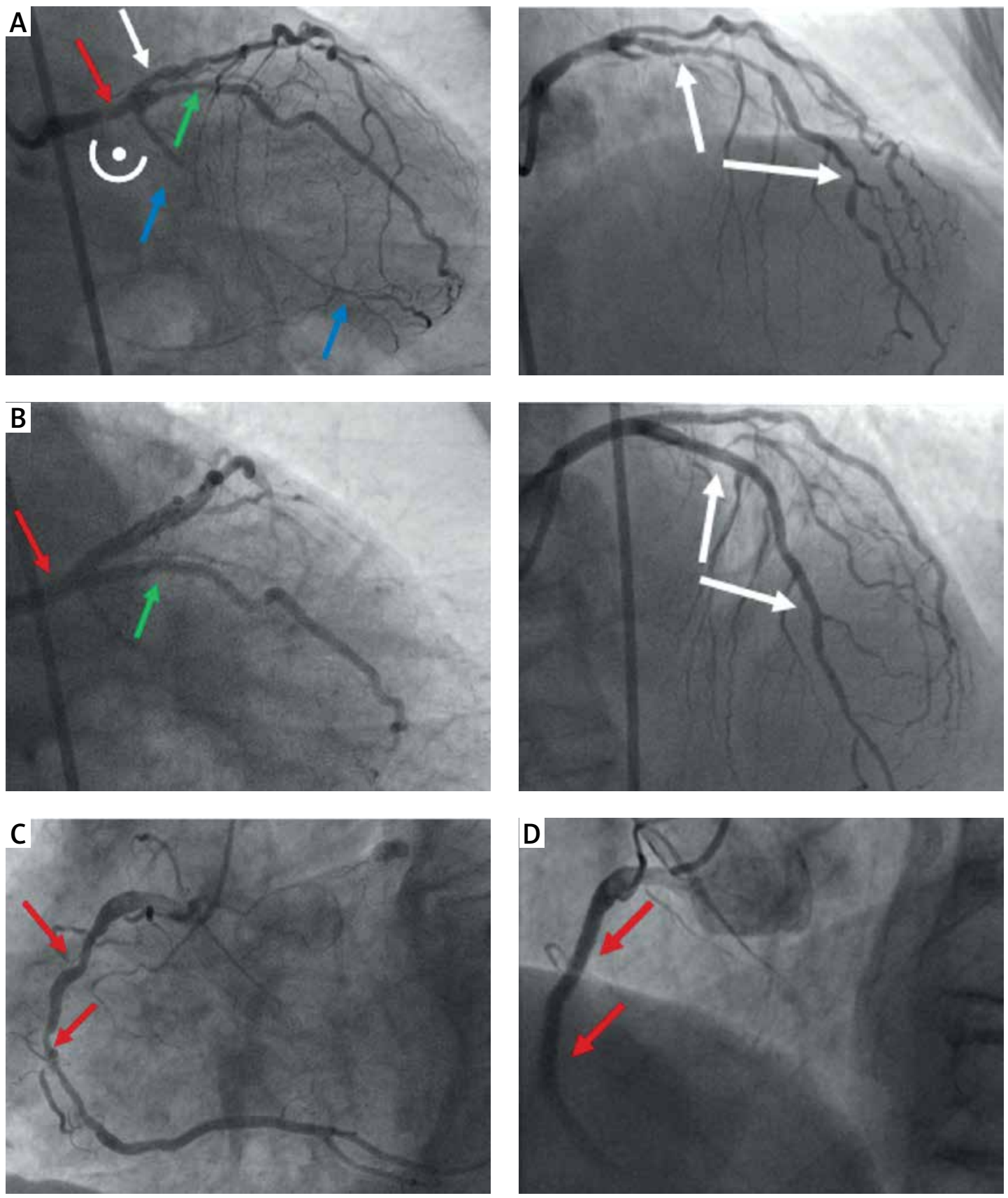

Figure 1. A - Coronary angiography showing $70 \%$ stenosis of the left main coronary artery (red arrow), diffuse significant lesions in the left anterior descending artery (white arrows), 60-70\% stenosis of the intermediate branch (green arrow) and chronic total occlusion of the circumflex artery in the middle segment (blue arrow), with collateral circulation from the left anterior descending artery and right coronary artery to the distal segment (blue arrow). B - Result of percutaneous coronary intervention with stent implantation to the left main coronary artery $(3.5 \times 23 \mathrm{~mm}$, red arrow), two overlapping stents to the left anterior descending artery $(3.0 \times 33 \mathrm{~mm}, 2.5 \times 33 \mathrm{~mm}$; white arrows) and a stent to the intermediate branch $(2.75 \times 12 \mathrm{~mm}$; green arrow). It was not feasible to insert the guidewire into the circumflex artery due to the origin angle $\left(\sim 90^{\circ}\right.$, curved white line). C - Coronary angiography showing diffuse lesions in the right coronary artery (red arrows) with a fractional flow reserve of 0.63 . D - Result of percutaneous coronary intervention with implantation of two overlapping stents to the right 
of the right femoral artery was diagnosed and treated with endovascular embolization.

The second stage of $\mathrm{PCl}$ was postponed until the clinical stabilization. Two EES $(3.5 \times 38 \mathrm{~mm}$; $4.0 \times 38 \mathrm{~mm}$ ) were implanted into the RCA via the right radial artery access (Figure $1 \mathrm{D}$ ). The anginal symptoms alleviated and the further course was uneventful, confirming the completeness of revascularization by clinical judgement [2]. Control echocardiography showed no new wall motion abnormalities and normal left ventricle ejection fraction. The two-year-follow-up after the procedures was uneventful.

In conclusion, there is a clear indication for CABG in patients with three-vessel MVD and a high SYNTAX score [1]. In case of the patient's refusal, $\mathrm{PCl}$ is a feasible alternative to achieve close-to-complete revascularization, yet challenging and associated with potential complications. First, wire manipulations in the LMCA may lead to thrombus formation and hemodynamic instability. Second, staged $\mathrm{PCI}$ in MVD often requires multiple access routes, increasing the risk of access site complications [3]. Third, multiple procedures with contrast administration are associated with contrast-induced acute kidney injury, which increases the risk of mortality [4]. Finally, the radiation burden due to repeated complex PCls should not be neglected. Careful planning, operator's experience and complication risk should all be taken into account when making the final decision regarding the treatment choice.

\section{Conflict of interest}

The authors declare no conflict of interest.

\section{References}

1. Neumann FJ, Sousa-Uva M, Ahlsson A, et al. 2018 ESC/ EACTS Guidelines on myocardial revascularization. Eur Heart J 2019; 40: 87-165.

2. De Innocentiis C, Zimarino M, De Caterina R. Is complete revascularisation mandated for all patients with multivessel coronary artery disease? Interv Cardiol 2018; 13: 45-50.

3. Kopin D, Seth M, Sukul D, et al. Primary and secondary vascular access site complications associated with percutaneous coronary intervention: insights from the BMC2 Registry. Cardiovasc Interv 2019; 12: 2247-56.

4. Brown JR, Malenka DJ, DeVries JT, et al. Transient and persistent renal dysfunction are predictors of survival after percutaneous coronary intervention: insights from the Dartmouth Dynamic Registry. Catheter Cardiovasc Interv 2008; 72: 347-54. 